Planetary Systems in the Universe - Observation, Formation and Evolution

Proceedings IAU Symposium No. 202, (c)2004 IAU

Alan Penny, Pawel Artymowicz, Anne-Marie Lagrange, $\mathcal{B}$ Sara Russell, eds.

\title{
Simulation and Modeling of Transit Eclipses by Planets
}

\author{
E.F. Milone. M.D. Williams, C.R. Stagg, M.L. McClure, \\ B. Desnoyers Winmill
}

Physics and Astronomy Dept, Univ. of Calgary, 2500 University Dr. N.W., Calgary, Alberta T2N-1N4, Canada

T. Brown

High Altitude Observatory, National Center for Atmospheric Research, P.O. Box 3000, Boulder, CO 80307-3000, USA

D. Charbonneau

Harvard-Smithsonian Center for Astrophysics, 60 Garden Street, Cambridge, MA 02138, USA

R.L. Gilliland

ST ScI, 3700 San Martin Dr., Baltimore, MD 21218, USA

G.W. Henry

Center of Excellence in Information Systems, Tennessee State Univ., 330 10th Ave. N., Nashville, TN 37203, USA

J. Kallrath

BASF-AG ZX/ZC Building C13 Ludwigshafen 67056, Germany

G.W. Marcy

Department of Astronomy, Univ. of California, Berkeley, CA USA

94720; Department of Physics and Astronomy, San Francisco, CA 94132, USA

Dirk Terrell

Southwest Research Institute, Suite 426, 1050 Walnut Street, Boulder, CO 80302-5143, USA

W. VanHamme

Florida International Univ., Dept. of Physics, Univ. Park, Miami, FL 33199, USA

\begin{abstract}
Light curve analysis by MDW of the photometry and RV data accumulated to date on HD 209458 has made use of a simulations database created for an 8-day HST observing project led by RLG to look for transits in 47 Tuc. We report progress in developing a consistent set of parameters obtained with our versions of the Wilson-Devinney program, WD98 and wd98k93, specially
\end{abstract}


modified to treat large grid sizes, corresponding to objects with radii exceeding $0.7 R_{J}$ and masses greater than $0.1 M_{J}$.

This work is supported in part by grants to EFM by Canadian NSERC and by the Univ. of Calgary Research Grants Committee.

\section{Introduction}

A July 1999, 8.3 day HST experiment (Taking the measure of Planets in the Globular Cluster 47 Tuc), organized and described by R. L. Gilliland, et al., (2000), was designed to search for transits by objects as small as Saturn in a densely crowded star field. The globular cluster 47 Tucanae, a rich, bright cluster, was selected to give the highest probability of finding transiting planets. The time-series data extraction, described by Brown, et al. (2000), has produced to present writing 75 variables. Only one of these seemed to offer promise as planetary transit candidate: WF2-V02, but this has now proven to be an artifact of the processing.

While 10's of planets were expected to be discovered in 47 Tuc by means of transit technique, the first discovery of a photometric planetary transit was reported instead by Henry, et al., (2000), and by Charbonneau, et al, (2000), following radial velocity work which identified a planet in this system. Consequently, Williams added the latter to his program of modeling based on planetary transit work carried out for the 47 Tuc project. As well as fitting model curves to data, predicted light curves in the infrared have also been produced by Williams. The density of the planet is found to be $399 \frac{\mathrm{kg}}{\mathrm{m}^{3}}$ and the planetary albedo was assumed to be 0.52 . The best results for each system are shown in table 1.

\section{Analysis Procedure}

The programs used for the analyses are the latest University of Calgary versions of the Wilson-Devinney program. WD98 and wd98k93d (see Milone, et al., 2000), developed over the past two years by Josef Kallrath from the WD95 package (Kallrath, et al., 1998; Kallrath \& Milone 1999), and the more recent base code provided by Wilson (1998). WD98 is a self-iterating damped-least squares package, with Kurucz atmospheric models, and with all the upgrades of Wilson's code.

The initial parameters for the HD209458 modeling came from Charbonneau, et al., (2000) and Henry, et al., (2000). The initial values of the mass, temperature, $M_{V}, \log \mathrm{g}$, and radius of the dominant star in the 47 Tuc eclipsing systems were obtained from the nearest 47 Tuc isochrone (D. VandenBerg, 1999) point beneath the CMD position of the system. Iterations were performed until the parameter corrections were exceeded by the m.s.e.s and where possible, the p.e.s. The final error of the best fit (the m.s.e. of a single observation of average weight) appears in the last column of the results, table 1. 


\section{Results}

Further examination of the data has shown the variability in WF2-V02 to be due to contamination from a saturated blue straggler variable, adjacent to it. The WF2-V02 results show that the search did have the sensitivity to find transits with a depth similar to that expected for giant planets. That the modeling largely ruled out the possibility of a planet in this case demonstrates the need for detailed modeling of suspected planetary transits.

The conclusion to be drawn from the 47 Tuc experiment is that there is a significant shortfall in the number of gas giant planets with periods less than a week in 47 Tucanae. There are two possible explanations: either higher metallicity is required for planetary formation; and/or such objects cannot survive the dynamical interactions in the cluster. In any case, there is an absence of 'hot Jupiters' in the fields surveyed in 47Tuc.

Table 1. $\mathrm{M}$ and $\mathrm{R}$ are in solar units except noted otherwise. $\Omega$ is the modified Roche potential. $\mathrm{T}$ is the mean effective temperature.

\begin{tabular}{c|c|c|c}
\hline \hline System & Star/Planet & HD 209458 & HD209458b \\
\hline System Elements & $\mathrm{P}$ (days) & & $3.524779(295)$ \\
& epoch $(245 . .)$. & & $1254.5857(148)$ \\
& $\mathrm{a}\left(R_{\odot}\right)$ & & $9.925(744)$ \\
& $\mathrm{i}$ & & $86.32(10)$ \\
& $\mathrm{q}=M_{2} / M_{1}$ & & $0.00059(2)$ \\
\hline Star/Planet Parameters & $\Omega$ & $8.772(41)$ & $1.5432(6)$ \\
& $\mathrm{M}$ & 1.06 & $0.655 M_{J}$ \\
& $\mathrm{R}$ & 1.13 & $1.27 R_{J}$ \\
& $\mathrm{~T}(\mathrm{~K})$ & $6037(70)$ & $998(531)$ \\
& $M_{\text {bol }}$ & 4.33 & 16.76 \\
\hline Fit Error & $\sigma_{1}(\mathrm{av}, \mathrm{wt})$ & & \pm 0.00153 \\
\hline
\end{tabular}

\section{References}

Brown, T.M., et al. 2000, BAAS, 32, 676

Charbonneau, D., Brown, T., Latham D., \& Mayor M. 2000, ApJ, 529, L45

Gilliland, R.L., et al. 2000, BAAS, 32, 675

Guhathakurta, P., et al. 2000, BAAS, 32, 740

Henry, G., Marcy, G.W., Butler, R.P., \& Vogt, S.S. 2000, ApJ, 529, L41

Howell, J.H., et al. 2000, BAAS, 32, 740

Kallrath, J. \& Milone, E.F. 1999, Eclipsing Binary Stars: Modeling and Analysis. (New York Springer-Verlag)

Kallrath, J., Milone, E.F., Terrell, D., \& Young, A.T. 1998, ApJ, 508, 308

Milone, E.F., et al. 2000, JRASC, in press

VandenBerg D. 1999, private communication

Wilson, R.E. 1998, Computing Binary Star Observables, (Gainesville Astronomy Dept. Univ of Florida) 